\title{
A physical solution for solving the zero-flow singularity in static ther- mal-hydraulics mixing models
}

\author{
Daniel Bouskela \\ Baligh El Hefni \\ EDF R\&D \\ 6, quai Watier F-78401 Chatou Cedex, France \\ daniel.bouskela@edf.fr baligh.el-hefni@edf.fr
}

\begin{abstract}
For the 0D-1D modelling of thermal-hydraulics systems, it is common practice to use static mixing models to compute the mixing specific enthalpy in fluid junctions such as mergers or splitters. However, this simplification leads to a well known singularity when the mass flow rate inside the junction goes to zero. The origin of the singularity is explained, and a rigorous physical solution is proposed to eliminate the singularity. A prototype implementation has been developed in the ThermoSysPro library for power plant modelling that illustrates the interest of the proposed solution, shows the impact on the structure of the library and enables to evaluate the computing overhead with respect to several possible variants.
\end{abstract}

Keywords: thermal-hydraulics; mixing models; convection; diffusion; ThermoSysPro

\section{Introduction}

When modelling thermal-hydraulics at the system level, such as power plants, it is common practice to use static equations to compute fluid quantities in mixing equipments such as mergers and splitters. This simplification stems from the fact that the volume of mixing is often neglected in junctions, therefore eliminating the differential term in the balance equations. It also occurs when computing isolated operating points that only require static models.

Neglecting diffusion is very common when one deals with large mass flow rates, as diffusion is only significant when mass flow rates approach zero. When diffusion is neglected, the only thermal phenomenon remaining in the model is convection. However when mass flows go to zero, convection disappears. So if diffusion is neglected, when mass flow rates go to zero, as convection also disappear, there is no thermal phenomena left in the model, leading to a possible indetermination of the enthalpy. This indetermination results in a singularity when static models are used, because in such case there is no differential variable to act as a memory for the enthalpy when mass flow rates are equal to zero.

In subsequent chapters, the mathematical origin of the singularity is explained. Then a rigorous mathematical formulation is proposed based on physical insight to remove the singularity. The idea is to reintroduce diffusion in static mixing models. Finally, a performance benchmark is given, based on a prototype implementation in ThermoSysPro. ThermoSysPro is a Modelica library developed by EDF for the modelling of power plants of all types [1].

\section{Computing the state of a thermal- hydraulics system}

As the objective is to find the origin of the physical singularity before giving a solution for removing the singularity, it is useful to understand how the physical state of a thermal-hydraulic system such as a volume is defined.

A volume is an abstract physical component where incoming flows mix. Figure 1 features four incoming flows. Flows are positive when they enter the volume and negative otherwise.

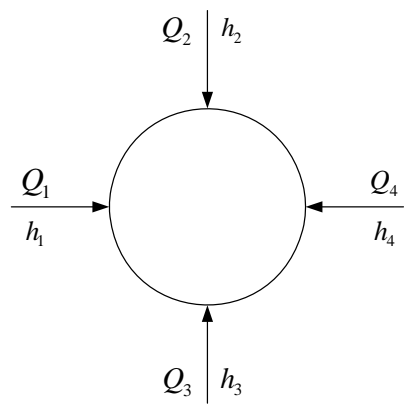

Figure 1: volume

In general, the state of a physical system is given by the set of independent physical quantities that completely define the state. There are many ways to choose the state variables for a given physical sys- 
tem. For a thermal-hydraulic volume $a$, a common choice is to use the average pressure $P_{a}$ and the average specific enthalpy $h_{a}$ inside the volume. Then the state of the volume $a$ will be defined if $P_{a}$ and $h_{a}$ can be computed.

In the sequel, we are only interested in computing $h_{a}$ which is called the mixing enthalpy in volume $a$.

To compute $h_{a}$, one must consider the neighboring volumes of $a$ which are collectively denoted $b$ (see Figure 2).

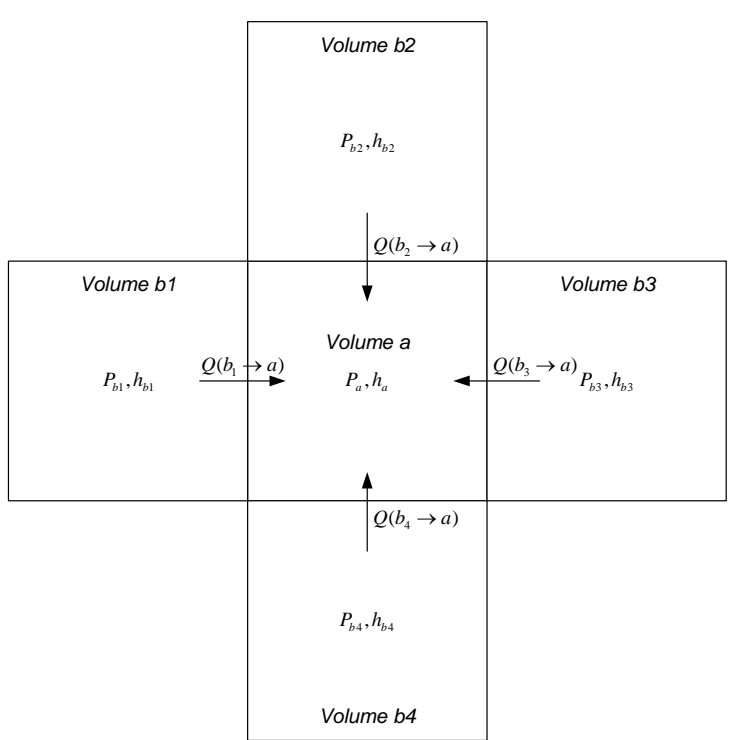

Figure 2: grid scheme

Each volume is assumed to be in thermodynamic equilibrium, so that their thermodynamic state is physically defined. However neighboring volumes may have different physical states, so that pressure and temperature gradients may exist that cause mass and energy flows between neighboring volumes through their common limiting boundary.

Mass flowing from volume $b$ to volume $a$ is denoted $Q(b \rightarrow a)$. Therefore $Q(b \rightarrow a)$ is positive if the flow actually occurs from $b$ to $a$, and negative otherwise. So $Q(a \rightarrow b)=-Q(b \rightarrow a)$.

Notice that the relation

$$
Q(a \rightarrow b)+Q(b \rightarrow a)=0
$$

is not a mass balance equation between volumes $b$ and $a$, but merely states the fact that $Q(b \rightarrow a)$ and $Q(a \rightarrow b)$ denote the same physical quantity with opposite sign conventions.
The specific enthalpy of flow $Q(b \rightarrow a)$ is denoted $h_{b: a}$. The meaning of notations is recalled in chapter 7.

The dynamic mass and energy balance equations are given by

$$
\begin{gathered}
\frac{d\left(\rho_{a} \cdot V_{a}\right)}{d t}=\sum_{b} Q(b \rightarrow a) \\
\frac{d\left(\rho_{a} \cdot V_{a} \cdot u_{a}\right)}{d t}=\sum_{b}\left(h_{b: a} \cdot Q(b \rightarrow a)+J(b \rightarrow a)\right)+W_{a}
\end{gathered}
$$

$J(b \rightarrow a)$ is the energy flow through diffusion.

$$
J(b \rightarrow a)=-A_{b: a} \cdot k_{b: a} \cdot \nabla T(b \rightarrow a)
$$

The static mass and energy balance equations are obtained by eliminating the dynamic terms on the left hand sides.

$$
\begin{gathered}
0=\sum_{b} Q(b \rightarrow a) \\
0=\sum_{b}\left(h_{b: a} \cdot Q(b \rightarrow a)+J(b \rightarrow a)\right)+W_{a}
\end{gathered}
$$

As the quantity $h_{a}$ does not appear explicitly in the static energy balance equation, it must be computed though the quantities $h_{b: a}$. So the relation between $h_{a}$ and $h_{b: a}$ must be established.

To that end, the fluid vein between volumes $a$ and $b$ is considered (see Figure 3).

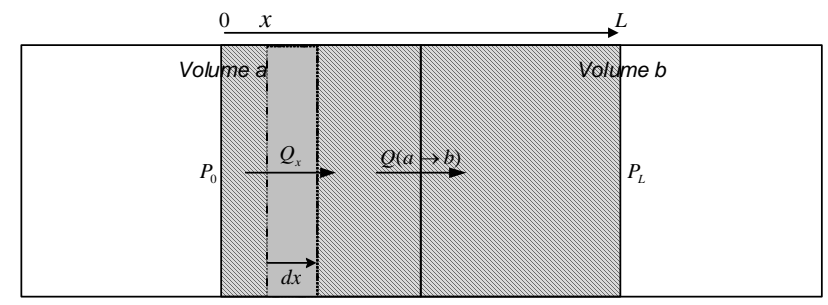

Figure 3: fluid vein

The static mass and energy balance equations in the volume limited by $d x$ is

$$
\begin{gathered}
0=\frac{\partial Q_{x}}{\partial x} \\
0=\frac{\partial}{\partial x}\left(h_{x} \cdot Q_{x}-A \cdot k \cdot \frac{\partial T_{x}}{\partial x}+W_{x}\right)
\end{gathered}
$$

Eq. (4) states that $Q_{x}$ is constant. Therefore:

$$
Q_{x}=Q(a \rightarrow b)=-Q(b \rightarrow a)
$$

In order to find an analytical solution to Eq. (5), the following relation between $d h_{x}$ and $d T_{x}$ is considered: 


$$
d h_{x}=c_{P} \cdot d T_{x}
$$

In the sequel, it is assumed that the relation given by Eq. (6) is valid (i.e. outside of the saturation line, for isobaric transformations, or for ideal gases, or when the contribution of the pressure variation to the variation of the specific enthalpy is negligible as compared to the contribution of the variation of temperature).

Under the additional assumption that $W_{x}=0$, the energy balance equation writes:

$$
0=Q(a \rightarrow b) \cdot \frac{\partial h_{x}}{\partial x}-\frac{A \cdot k}{c_{p}} \cdot \frac{\partial^{2} h_{x}}{\partial x}
$$

Eq. (7) can be solved analytically [2]:

$$
h_{x}=\frac{1}{1-e^{P_{e}}} \cdot\left[h_{b}-h_{a} \cdot e^{P_{e}}+\left(h_{a}-h_{b}\right) \cdot e^{P_{e} \cdot \frac{x}{L}}\right]
$$

with

$$
\begin{gathered}
P_{e}=\frac{1}{\gamma} \cdot Q(a \rightarrow b) \\
\gamma=\frac{A \cdot k}{C_{P} \cdot L}
\end{gathered}
$$

$h_{b: a}$ is the value of $h_{x}$ for $x=\frac{L}{2}$ :

$$
h_{b: a}=h_{L / 2}=\widehat{s}\left(P_{e}\right) \cdot h_{a}+\widehat{s}\left(-P_{e}\right) \cdot h_{b}
$$

with

$$
\widehat{s}(x)=\frac{1}{1+e^{-\frac{x}{2}}}
$$

Figure 4 gives a plot of $\hat{s}$.

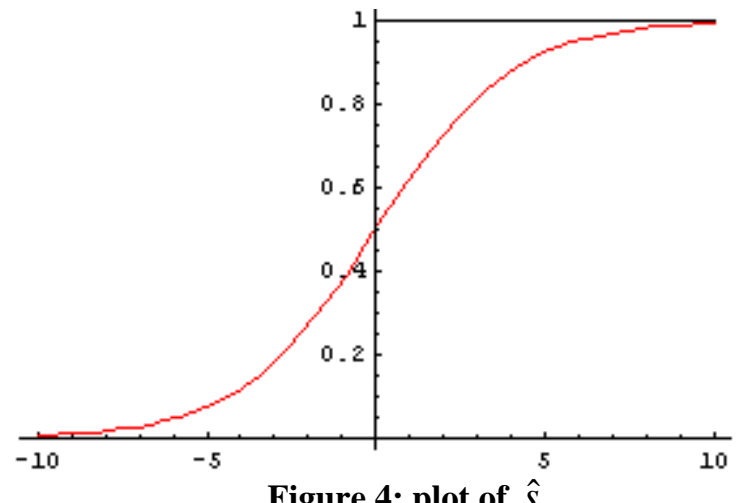

Figure 4: plot of $\hat{s}$

If diffusion is neglected, then $\gamma \rightarrow 0, P_{e} \rightarrow+\infty$ and $h_{b: a}$ becomes:

$$
h_{b: a}=s(Q(a \rightarrow b)) \cdot h_{a}+s(Q(b \rightarrow a)) \cdot h_{b}
$$

$$
s(x)=\left\{\begin{array}{lll}
1 & \text { if } & x>0 \\
\frac{1}{2} & \text { if } & x=0 \\
0 & \text { if } & x<0
\end{array}\right.
$$

This is the well known upwind scheme approximation for flow reversal. This relation is widely used, even if the assumptions used in this derivation are not fulfilled.

Note that $S$ is discontinuous at $x=0$, whereas $\hat{S}$ is continuous and differentiable everywhere.

\section{Origin of the singularity in static mixing models}

The objective of this chapter is to show that the singularity in static mixing models arises when diffusion is neglected.

So in the sequel diffusion is neglected, which means that $J(b \rightarrow a)=0$. Also $Q(b \rightarrow a)$ is denoted $Q_{b}$ to simplify the notation.

The mass and energy balance equations become

$$
\begin{gathered}
0=\sum_{b} Q_{b} \\
0=\sum_{b} h_{b: a} \cdot Q_{b}
\end{gathered}
$$

The value of the enthalpy $h_{b: a}$ is given by the upwind scheme (see Eq. (13)):

$$
h_{b: a}=s\left(Q_{b}\right) \cdot h_{b}+s\left(-Q_{b}\right) \cdot h_{a}
$$

In the sequel, the following relations are used:

$$
\begin{gathered}
|x|=\operatorname{sgn}(x) \cdot x \\
\operatorname{sgn}(x)=s(x)-s(-x) \\
s(x)=1-s(-x)
\end{gathered}
$$

where sgn is the sign function.

Then using Eq. (15), (16), (17) and (20)

$$
\begin{aligned}
& \sum_{b} h_{b: a} \cdot Q_{b}=\sum_{b} s\left(Q_{b}\right) \cdot Q_{b} \cdot h_{b}+h_{a} \cdot \sum_{b} s\left(-Q_{b}\right) \cdot Q_{b} \\
& =\sum_{b} s\left(Q_{b}\right) \cdot Q_{b} \cdot h_{b}+h_{a} \cdot\left(\sum_{b} Q_{b}-\sum_{b} s\left(Q_{b}\right) \cdot Q_{b}\right) \\
& =\sum_{b} s\left(Q_{b}\right) \cdot Q_{b} \cdot h_{b}-h_{a} \cdot \sum_{b} s\left(Q_{b}\right) \cdot Q_{b}=0
\end{aligned}
$$

Therefore

$$
h_{a}=\frac{\sum_{b} s\left(Q_{b}\right) \cdot Q_{b} \cdot h_{b}}{\sum_{b} s\left(Q_{b}\right) \cdot Q_{b}}
$$


when $\sum_{b} s\left(Q_{b}\right) \cdot Q_{b} \neq 0$.

To find out when this condition is satisfied, using Eq. (15), (18), (19) and (20):

$\sum_{b}\left|Q_{b}\right|=\sum_{b} \operatorname{sgn}\left(Q_{b}\right) \cdot Q_{b}=\sum_{b}\left(s\left(Q_{b}\right)-s\left(-Q_{b}\right)\right) \cdot Q_{b}$ $=\sum_{b} s\left(Q_{b}\right) \cdot Q_{b}-\sum_{b} s\left(-Q_{b}\right) \cdot Q_{b}$

$=\sum_{b} s\left(Q_{b}\right) \cdot Q_{b}-\sum_{b}\left(1-s\left(Q_{b}\right)\right) \cdot Q_{b}$

$=2 * \sum_{b} s\left(Q_{b}\right) \cdot Q_{b}-\sum_{b} Q_{b}=2 * \sum_{b} s\left(Q_{b}\right) \cdot Q_{b}$

Hence:

$$
h_{a}=2 \cdot \frac{\sum_{b} s\left(Q_{b}\right) \cdot Q_{b} \cdot h_{b}}{\sum_{b}\left|Q_{b}\right|}
$$

when $\sum_{b}\left|Q_{b}\right| \neq 0$.

So when all mass flow rates are equal to zero, the mixing enthalpy $h_{a}$ is indeterminate $\left(h_{a}=0 / 0\right)$.

Although the indetermination occurs only at an isolated point (all mass flow rates equal to zero), it is not obvious to extend $h_{a}$ in order to remove the singularity at zero (contrary to other functions with isolated singularities such as $\sin (x) / x)$.

In particular, it is not sufficient to replace $s$ by $\hat{s}$ (or in other words get rid of the upwind scheme by introducing diffusion in the flow reversing formula given by Eq. (17)) because then

$$
h_{a}=\frac{\sum_{b} \hat{s}\left(\hat{Q}_{b}\right) \cdot Q_{b} \cdot h_{b}}{\sum_{b} \hat{s}\left(\hat{Q}_{b}\right) \cdot Q_{b}}
$$

with

$$
\hat{Q}_{b}=\frac{Q_{b}}{\gamma_{b: a}}
$$

The singularity still remains since $\sum_{b} \hat{s}\left(\hat{Q}_{b}\right) \cdot Q_{b}=0$ when all mass flow rates are equal to zero.

However, noticing that

$$
s\left(Q_{b}\right) \cdot Q_{b}=\max \left(Q_{b}, 0\right)
$$

Eq. (21) may be written as

$$
h_{a}=\frac{\sum_{Q_{b}>0} Q_{b} \cdot h_{b}}{\sum_{Q_{b}>0} Q_{b}}
$$

Therefore, if one is not interested in the correct value of $h_{a}$ near zero flows, which is in general the case when diffusion is neglected, then as suggested in [3] one can replace $Q_{b}$ by $\max \left(Q_{b}, Q_{\varepsilon}\right)$ where $Q_{\varepsilon}$ is a small positive mass flow rate. Then when all mass flow rates $Q_{b}$ are below $Q_{\varepsilon}\left(\left|Q_{b}\right|<Q_{\varepsilon}\right)$ :

$$
h_{a}=\frac{1}{N_{a}} \sum_{b} h_{b}
$$

where $N_{a}$ is the number of neighboring volumes $b$ of volume $a$, so the singularity is removed for zero flows.

Noticing that $\max (x, y)=s(x-y) \cdot(x-y)+y$, one can even have a $C^{\infty}$ way of removing the singularity by (1) considering the function

$$
s_{p}(x)=\frac{1}{1+e^{-p \cdot x}}
$$

by (2) replacing $s$ by $s_{p}$ in the max function above

$$
\operatorname{smoothMax}(x, y, p)=s_{p}(x-y) \cdot(x-y)+y
$$

and by (3) properly adjusting the value of $p$ wrt. $Q_{\varepsilon}$.

This solution will not be developed here any further, as a full physical solution is sought. The reason is that replacing $Q_{b}$ by $Q_{\varepsilon}$ when $\left|Q_{b}\right|<Q_{\varepsilon}$ for computing the mixing enthalpy violates the energy balance fundamental law, so requires a proper choice of $Q_{\varepsilon}$ wrt. the problem at hand.

As suggested in [3] there are also other ways to write smoothMax.

Notice also that $\hat{s}=s_{0.5}$ and $s=s_{\infty}$ (see Eq. (12), (14) and (26).

\section{Removing the singularity at zero flows}

Diffusion is reinstalled in the energy balance equation. Then

$$
\begin{gathered}
0=\sum_{b} Q(b \rightarrow a) \\
0=\sum_{b}\left(h_{b: a} \cdot Q(b \rightarrow a)+J(b \rightarrow a)\right)
\end{gathered}
$$

assuming without loss of generality that $W_{a}=0$.

Using Eq. (1) and (6): 


$$
\begin{aligned}
& J(b \rightarrow a)=(A \cdot k)_{b: a} \cdot\left(\frac{\partial T_{x}}{\partial x}\right)_{L / 2} \\
& =\left(\frac{A \cdot k}{C_{p}}\right)_{b: a} \cdot\left(\frac{\partial h_{x}}{\partial x}\right)_{L / 2}
\end{aligned}
$$

Taking the derivative of Eq. (8) wrt. $x$ at $x=\frac{L}{2}$ yields:

$$
J(b \rightarrow a)=r\left(P_{e}\right) \cdot J_{0}(b \rightarrow a)
$$

with

$$
\begin{gathered}
J_{0}(b \rightarrow a)=\gamma_{b: a} \cdot\left(h_{b}-h_{a}\right) \\
\gamma_{b: a}=\left(\frac{A \cdot k}{c_{P} \cdot L}\right)_{b: a} \\
P_{e}=-\frac{1}{\gamma_{b: a}} \cdot Q(b \rightarrow a) \\
r(x)= \begin{cases}\frac{x}{e^{\frac{x}{2}}-e^{-\frac{x}{2}}} \text { if } x \neq 0 \\
1 \quad \text { if } x=0\end{cases}
\end{gathered}
$$

$J_{0}(b \rightarrow a)$ is the energy flux when $Q(b \rightarrow a)=0$.

For easier computation $r(x)$ may be approximated by the Gaussian

$$
\widehat{r}(x)=e^{-0.033 \cdot x^{2}}
$$

The plot below compares $r$ in red with $\hat{r}$ in blue.

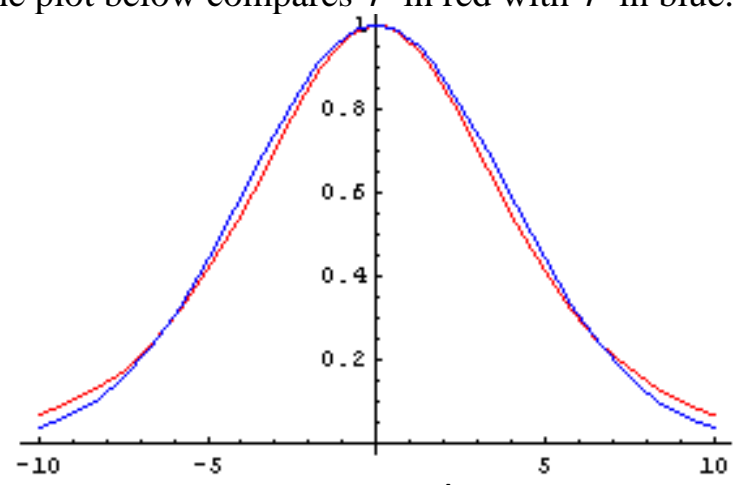

Figure 5: plot of $\hat{r}$ and $r$

When all flows $Q_{b}$ are equal to zero, the energy balance equation writes

$$
0=\sum_{b} J_{0}(b \rightarrow a)=\sum_{b} \gamma_{b: a} \cdot\left(h_{b}-h_{a}\right)
$$

As the coefficients $\gamma_{b: a}$ are always strictly positive $\left(\gamma_{b: a}>0\right)$, when all flows are equal to zero the mixing enthalpy $h_{a}$ is defined and takes the value:

$$
h_{a}=\frac{\sum_{b} \gamma_{b: a} \cdot h_{b}}{\sum_{b} \gamma_{b: a}}
$$

If all coefficients $\gamma_{b: a}$ are equal, then $h_{a}$ is the arithmetic mean (see also Eq. (25))

$$
h_{a}=\frac{1}{N_{a}} \sum_{b} h_{b}
$$

where $N_{a}$ is the number of neighboring volumes $b$ of volume $a$.

As a conclusion to this chapter, when diffusion is taken into account, the energy balance equation is

$$
0=\sum_{b}\left(h_{b: a} \cdot Q(b \rightarrow a)+\left(h_{b}-h_{a}\right) \cdot Q_{\varepsilon}(b \rightarrow a)\right)
$$

with

$$
Q_{\varepsilon}(b \rightarrow a)=r\left(-\frac{1}{\gamma_{b: a}} \cdot Q(b \rightarrow a)\right) \cdot \gamma_{b: a}
$$

The terms $Q_{\varepsilon}(b \rightarrow a)$ are in general small but are always strictly positive and have the same physical unit as a mass flow rate $(\mathrm{kg} / \mathrm{s})$. So they never go to zero, even when all mass flow rates go to zero. They act therefore as small positive mass flow rates that remove naturally in a $C^{\infty}$ way the singularity of the mixing enthalpy at zero flows.

\section{Benchmark of the proposed solu- tion}

To evaluate the computing overhead of introducing diffusion to solve the singularity problem, the benchmark consists in comparing two alternatives for the static energy balance equation (see Eq. (28)):

- Alternative 1: without diffusion, with or without upwind scheme.

- Alternative 2: with diffusion, with or without upwind scheme.

Without diffusion means that

$$
J(b \rightarrow a)=0
$$

With diffusion means that

$$
J(b \rightarrow a)=\hat{r}\left(\hat{Q}_{b}\right) \cdot \gamma_{b: a} \cdot\left(h_{b}-h_{a}\right)
$$

With upwind scheme means that

$$
h_{b: a}=s\left(Q_{b}\right) \cdot h_{b}+s\left(-Q_{b}\right) \cdot h_{a}
$$

Without upwind scheme means that

$$
h_{b: a}=\hat{s}\left(\hat{Q}_{b}\right) \cdot h_{b}+\hat{s}\left(-\hat{Q}_{b}\right) \cdot h_{a}
$$


$\hat{r}\left(\hat{Q}_{b}\right)$ and $\hat{s}\left(\hat{Q}_{b}\right)$ are defined by Eq. (12), (24) and (34).

The equations are implemented as a prototype in the ThermoSysPro library using the scheme shown in Figure 6.

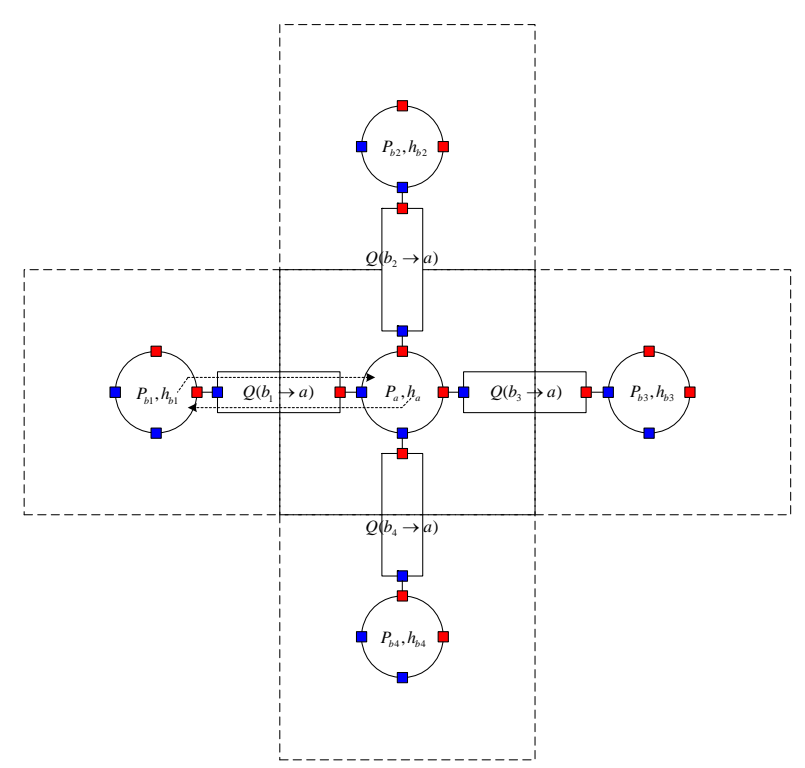

Figure 6: grid scheme in ThermoSysPro

The quantities $\left(P_{a}, P_{b}\right)$ and $\left(h_{a}, h_{b}\right)$ are computed in the multi-port elements with the mass and energy balance equations. Multi-port elements represent the control volumes $a$ and $b$. For this reason, they are also called 'volumes'. The quantities $J(b \rightarrow a)$ and $h_{b: a}$ are also computed in the volumes.

The quantities $Q(b \rightarrow a)$ are computed in the two-port elements with the momentum balance equations. Two-port elements represent the interfaces $b: a$ between $a$ and $b$.

The interface $b: a$ is oriented positively from the blue port to the red port of the two-port element that represents $b: a$. So the mass flow rate is positive when the fluid flows along the positive direction of the interface orientation, i.e. from the blue port to the red port. To reflect this sign convention for mass flow rates, the blue port is called 'input port', and the red port is called 'output port'.

The components are connected together via the input and output ports that correspond to Modelica connectors. Input and output connectors have the same structure. In order to handle diffusion, they are somewhat different from the usual fluid connectors used in Modelica fluid libraries, and in particular in the current distribution of the ThermoSysPro library. The meaning of the variables in the connector de- pends on whether the connector is attached to a volume or to a two-port element.

If the connector is attached to a two-port element representing the interface $b: a$ :

\begin{tabular}{|l|l|}
\hline P & $\begin{array}{l}\text { Pressure } P_{a} \text { in volume } a \text {, or pressure } P_{b} \text { in } \\
\text { volume } b \text {, depending on whether the connec- } \\
\text { tor is on the side of } a \text { or on the side of } b .\end{array}$ \\
\hline Q & $\begin{array}{l}\text { Mass flow rate } Q(b \rightarrow a) \text { of the fluid going } \\
\text { through interface } b: a .\end{array}$ \\
\hline h & $\begin{array}{l}\text { Specific enthalpy } h_{b: a} \text { of the fluid going } \\
\text { through interface } b: a .\end{array}$ \\
\hline h_vol_1 & $\begin{array}{l}\text { Specific enthalpy } h_{b} \text { or } h_{a} \text { of the fluid in } \\
\text { volume } b \text { or } a \text { located on the side of the } \\
\text { input port of the two-port element that } \\
\text { represents } b: a .\end{array}$ \\
\hline h_vol_2 & $\begin{array}{l}\text { Specific enthalpy } h_{b} \text { or } h_{a} \text { of the fluid in } \\
\text { volume } b \text { or } a \text { located on the side of the } \\
\text { output port of the two-port element that } \\
\text { represents } b: a .\end{array}$ \\
\hline
\end{tabular}

If the connector is attached to a multi-port element that represents volume $b$, and the connector is connected to the two-port element that represents interface $b: a$ :

\begin{tabular}{|c|c|}
\hline $\mathrm{P}$ & Pressure $P_{b}$ of the fluid in volume $b$. \\
\hline Q & $\begin{array}{l}\text { Mass flow rate } Q(b \rightarrow a) \text { of the fluid going } \\
\text { through interface } b: a \text {. }\end{array}$ \\
\hline $\mathrm{h}$ & $\begin{array}{l}\text { Specific enthalpy } h_{b: a} \text { of the fluid going } \\
\text { through interface } b: a \text {. }\end{array}$ \\
\hline h_vol_1 & $\begin{array}{l}\text { If the connector is an input port: specific en- } \\
\text { thalpy } h_{b} \text { of the fluid in the neighboring vo- } \\
\text { lume } b \text { located in the direction of negative } \\
\text { flow rates. } \\
\text { If the connector is an output port: specific } \\
\text { enthalpy } h_{a} \text { of the fluid in volume } a \text {. }\end{array}$ \\
\hline h_vol_2 & $\begin{array}{l}\text { If the connector is an input port: specific en- } \\
\text { thalpy } h_{a} \text { of the fluid in volume } a \text {. } \\
\text { If the connector is an output port: specific } \\
\text { enthalpy } h_{b} \text { of the fluid in the neighboring } \\
\text { volume } b \text { located in the direction of positive } \\
\text { flow rates. }\end{array}$ \\
\hline
\end{tabular}


The purpose of $h$ _vol_1 and $h$ _vol_2 is to provide both $h_{a}$ and $h_{b}$ to volumes $a$ and $b$ even if they are separated by a line of connected two-port elements.

When connecting together two connectors, the variables inside the connectors are made equal because they represent the same physical quantities. So connectors are used to assemble the model from the different components, and not to generate extra physical equations (such as balance equations for instance).

This scheme for distributing the equations between multi-port and two-port elements and connecting them together enables to connect together several two-port elements without having to separate them by volumes. The connected line of two-port elements is then equivalent to a single two-port element. Also, there are no infinitesimally small volume elements implied between two connected two-port elements, so the connections do not generate the kind of singularity dealt with in this paper.

The test model is shown in Figure 7.

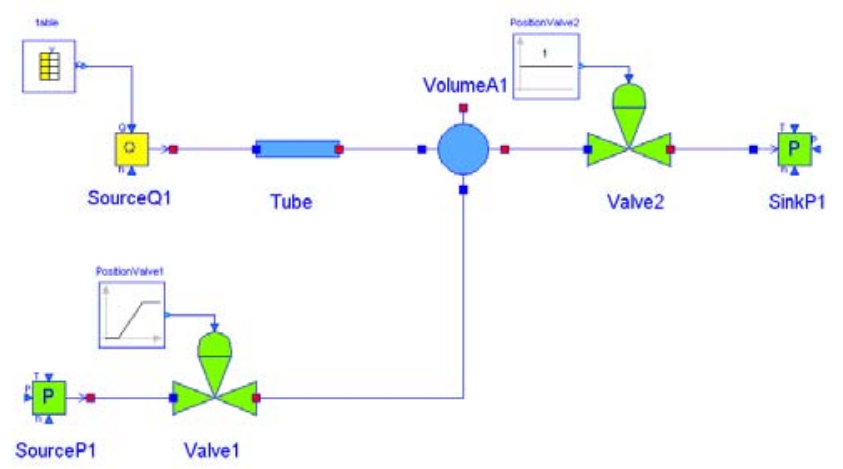

Figure 7: test model

A mixing volume (VolumeA1) is connected to two fluid sources (SourceQ1, SourceP1) and a fluid sink (SinkP1) via a pipe (Tube) and two control valves (Valve1 and Valve2). The test scenario consists in performing a flow reversal, and then setting all mass flow rates to zero.

\begin{tabular}{|l|l|}
\hline SourceQ1 & $\begin{array}{l}\text { The specific enthalpy is constant equal to } \\
1 . e 5 \mathrm{~J} / \mathrm{kg} . \\
\text { The mass flow rate follows the following } \\
\text { curve }(\mathrm{kg} / \mathrm{s} \text { vs. } \mathrm{s}) .\end{array}$ \\
\hline
\end{tabular}

\begin{tabular}{|l|l|}
\hline & \\
\hline SourceP1 & $\begin{array}{l}\text { The specific enthalpy is constant equal to } \\
1 . e 5 \mathrm{~J} / \mathrm{kg} . \\
\text { The pressure is constant equal to } 3 \text { bars. }\end{array}$ \\
\hline SinkP1 & $\begin{array}{l}\text { The temperature is constant equal to } 320 \mathrm{~K} \\
\text { The pressure is constant equal to } 1 \text { bar. }\end{array}$ \\
\hline Valve1 & $\begin{array}{l}\text { The position varies from } 100 \% \text { to } 0 \% \text { in } 2 \\
\text { seconds starting from } \mathrm{t}=1 \mathrm{~s} \text {. }\end{array}$ \\
\hline Valve2 & The position is constant equal to $100 \%$ \\
\hline
\end{tabular}

Four simulation runs are performed:

- Run 1.1: without diffusion, with upwind scheme

- Run 1.2: without diffusion, without upwind scheme

- Run 2.1: with diffusion, with upwind scheme

- Run 2.2: with diffusion, without upwind scheme

For each run are plotted:

- The mass flow rates at each connected port of the mixing volume (3 curves)

- The specific enthalpy inside the mixing volume (1 curve)

- The specific enthalpies inside each source and sink (3 curves).

Figure 8 gives the mass flow rates for all runs (no difference in results for all runs).

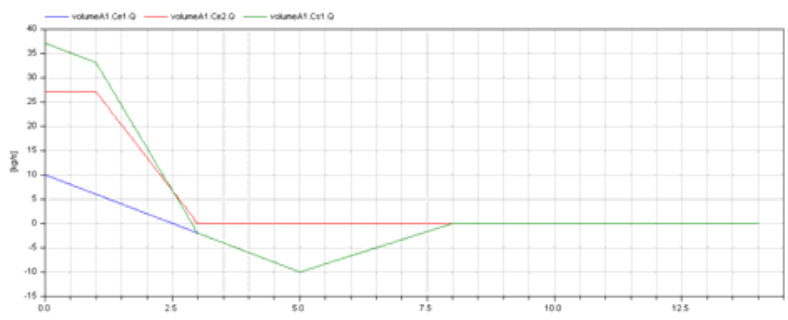

Figure 8: mass flow rates for runs 1 and 2 


\section{Runs 1.1 and 1.2}

Figure 9 gives the specific enthalpies for run 1.1, and Figure 10 gives the specific enthalpies for run 1.2.

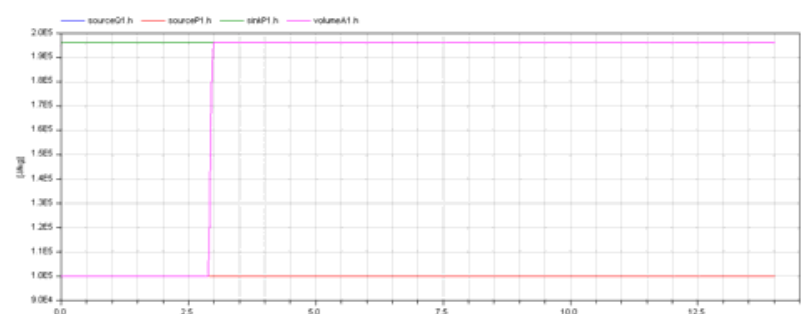

Figure 9: specific enthalpies for run 1.1

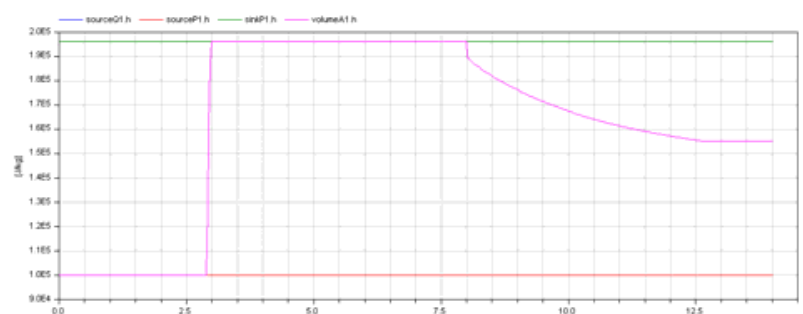

Figure 10: specific enthalpies for run 1.2

For run 1.1, when all mass flow rates are set to zero (at $t=8 \mathrm{~s}$ ), the specific enthalpy in the mixing volume keeps its last value prior to the zero mass flow rates condition, just as though there were some kind of memory holding this value when all mass flow rates become zero. This is probably an artifact due to the numerical methods used to solve the algebraic equations. The result is physically correct, but this looks as sheer luck as the theory predicts that the result is in fact mathematically undefined when diffusion is neglected.

To the contrary, for run 1.2, when all mass flow rates are set to zero (at $t=8 \mathrm{~s}$ ), the specific enthalpy in the mixing volume continues to vary until it takes a seemingly final constant value. This is a false transient which is of course unphysical because, since the model is static, all values should stay constant when the boundary conditions are constant (after $\mathrm{t}=$ $8 \mathrm{~s})$.

In both cases, the theory predicts that the mixing enthalpy can take any value when all mass flow rates are zero and diffusion is neglected, so the result is consistent with the theory.

\section{Runs 2.1 and 2.2}

Figure 11 gives the specific enthalpies for runs 2.1 and 2.2 (no difference in results for both runs).

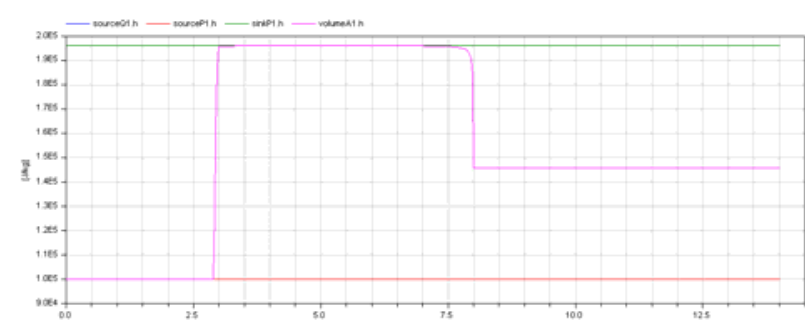

Figure 11: specific enthalpies for runs 2.1 and 2.2

When all mass flow rates are set to zero, the specific enthalpy in the mixing volume takes the value that corresponds to the thermal equilibrium between the mixing and the sources and sink it is connected with, which is a correct physical result. The transition to thermal equilibrium is sharp but continuous.

The following table gives the computing times in seconds and the sizes of the non-linear systems after manipulation for each run with Dymola.

\begin{tabular}{|c|l|c|}
\hline Run & CPU time & $\begin{array}{c}\text { Sizes of non-linear } \\
\text { systems }\end{array}$ \\
\hline 1.1 & 0.125 & $\{3,1\}$ \\
\hline 1.2 & 1.22 & $\{3,1\}$ \\
\hline 2.1 & 0.219 & $\{4\}$ \\
\hline 2.2 & 0.313 & $\{7\}$ \\
\hline
\end{tabular}

The conclusion from this experiment is that the best solution is to take into account diffusion in the energy balance equation, but still use the upwind scheme, i.e. neglect diffusion in the flow reversal equation. The overhead over the standard approximation of neglecting diffusion everywhere is $75 \%$.

More diverse experiments should be made in order to decide whether it is better to take into account diffusion in the flow reversal formula or not, because avoiding the upwind scheme enables to remove the discontinuity due to the use of the step function.

\section{Conclusion}

Neglecting diffusion in thermal-hydraulics systems is a common approximation when dealing with large mass flow rates, as diffusion is only significant when mass flow rates are near zero.

However, this approximation leads to undefined values for the mixing enthalpies when all mixing mass flow rates are equal to zero. This is due to the fact that convection, which is the only thermal phenomena taken into account when diffusion is neglected, vanishes when mass flow rates go to zero, so there is no physical phenomenon left to describe the thermal physical state inside the mixing volume. 
A rigorous mathematical and physical solution to this problem is to reinstall diffusion in the energy balance equation. This solution indeed removes the singularity for zero flows in a continuously differentiable way, as theoretically demonstrated in this paper.

A prototype implementation has been made in the ThermoSysPro library for power plant modelling, developed by EDF. The introduction of diffusion into the library has an impact on the structure of connectors.

The prototype has been tested on a small static model that features a mixing volume connected to fluid sources. The test scenario consists in performing a flow reversal, then bringing all flows to zero. The results are consistent with the theory developed in this paper. They also show that the upwind scheme, which is the equation for computing flow reversal that neglects diffusion, can be kept, as reinstalling diffusion in the flow reversal equation as well does not make any difference in the computing results, but provokes a significant overhead in computing time. However, more numerical experiments should be made to confirm this last point.

\section{Notations}

$P_{a}$ : fluid pressure in volume $a$

$h_{a}$ : fluid specific enthalpy in volume $a$

$u_{a}$ : fluid specific internal energy in volume $a$

$\rho_{a}$ : fluid density in volume $a$

$V_{a}$ : volume of volume $a$

$W_{a}$ : external energy brought to the volume $a$

$Q(b \rightarrow a)$ : mass flow rate of the fluid flowing from volume $b$ to volume $a$

$\nabla T(b \rightarrow a)$ : temperature gradient from volume $b$ to volume $a$

$h_{b: a}$ : specific enthalpy of the fluid flowing from volume $b$ to volume $a$

$\left(c_{p}\right)_{b: a}$ : specific heat capacity of the fluid flowing from volume $b$ to volume $a$

$k_{b: a}$ : diffusion coefficient at the interface between volumes $b$ and $a$

$A_{b: a}$ : area of the interface between volumes $b$ and $a$

$L_{b: a}$ : distance between the centers of volumes $b$ and $a$
$G_{b: a}$ : more generally, value of quantity $G$ at the interface between volumes $b$ and $a$

\section{Acknowledgments}

This work was partially supported by DGCIS within the ITEA 2 EUROSYSLIB project.

\section{References}

[1] El Hefni B., Bouskela D., Lebreton G., 'Dynamic modelling of a combined cycle power plant with ThermoSysPro', Modelica 2011 conference proceedings.

[2] Patankar S.V., 'Numerical Heat Transfer and Fluid Flow', Hemisphere Publishing Corporation, Taylor \& Francis, 1980.

[3] Franke R., Casella F., Otter M., Sielemann M., Elmqvist H., Mattson S.E., Olsson H., 'Stream Connectors - An extension of Modelica for Device-Oriented Modeling of Convective Transport Phenomena', Modelica 2009 conference proceedings. 